\title{
Acute motor and sensory axonal neuropathy in association with primary Sjögren's syndrome: a case report
}

\author{
Yu-Ming Chen ${ }^{1,2^{*}}$ and Kuei-Ying Su, 2,3
}

\begin{abstract}
Background: Primary Sjögren's syndrome is a chronic, autoimmune, connective tissue disorder that results from the infiltration of exocrine glands, especially the lacrimal and salivary glands, by autoantibodies. Patients with Sjögren's syndrome commonly present with dry eyes (xerophthalmia) and dry mouth (xerostomia). However, the clinical manifestations of Sjögren's syndrome can be complicated and variable due to involvement of extraglandular organ systems, such as the nervous system. The neurological manifestations of this disorder often precede those of other exocrine gland symptoms. Hence, early diagnosis of Sjögren's syndrome remains a challenge.

Case presentation: We report the case of a 63-year-old woman with primary Sjögren's syndrome who presented with acute motor and sensory axonal neuropathy (AMSAN). Treatment with glucocorticoids and immunosuppressants partially improved her muscle weakness and paresthesia.

Conclusions: This case demonstrates the importance of early recognition and diagnosis of AMSAN in association with primary Sjögren's syndrome to achieve a favorable clinical outcome. Primary Sjögren's syndrome may be underdiagnosed because of vague symptoms of the sicca complex. Comprehensive immunological testing to evaluate this condition may be performed in patients presenting with variants of Guillain-Barré syndrome.
\end{abstract}

Keywords: Acute motor and sensory axonal neuropathy, Guillain-Barré syndrome, Sjögren's syndrome

\section{Background}

Primary Sjögren's syndrome (pSS) is a chronic autoimmune disease characterized by the presence of autoantibodies to Sjögren's syndrome A (SSA) and SSB antigens with systemic manifestations reflecting the organs affected. Patients with pSS typically present with dysfunction of the exocrine glands, predominantly the lacrimal and salivary glands, in the form of dryness of the eyes (xerophthalmia) and mouth (xerostomia). However, this disease can also affect non-exocrine organ systems and cause various clinical manifestations. It can

\footnotetext{
* Correspondence: yumingchenmd@gmail.com

'Department of Neurology, Hualien Tzu Chi Hospital, Buddhist Tzu Chi Medical Foundation, Hualien, Taiwan

${ }^{2}$ School of Medicine, Tzu Chi University, Hualien, Taiwan

Full list of author information is available at the end of the article
}

also affect the central and peripheral nervous systems [1-3]. Neurological manifestations often precede exocrine gland symptoms such as xerophthalmia and xerostomia [4-6].

Here, we report the case of a 63-year-old woman who presented with fulminant and acute motor and sensory axonal neuropathy (AMSAN) and was subsequently diagnosed with pSS. She received immunosuppressive therapy and achieved partial improvement of the neurological deficits.

\section{Case presentation}

The patient was a 63-year-old woman with a history of adenomyosis, gout, and essential hypertension. She could previously perform basic activities of daily living (BADL) independently. Insidiously, she became symptomatic

(c) The Author(s). 2021 Open Access This article is licensed under a Creative Commons Attribution 4.0 International License, which permits use, sharing, adaptation, distribution and reproduction in any medium or format, as long as you give appropriate credit to the original author(s) and the source, provide a link to the Creative Commons licence, and indicate if changes were made. The images or other third party material in this article are included in the article's Creative Commons licence, unless indicated otherwise in a credit line to the material. If material is not included in the article's Creative Commons licence and your intended use is not permitted by statutory regulation or exceeds the permitted use, you will need to obtain permission directly from the copyright holder. To view a copy of this licence, visit http://creativecommons.org/licenses/by/4.0/ The Creative Commons Public Domain Dedication waiver (http://creativecommons.org/publicdomain/zero/1.0/) applies to the data made available in this article, unless otherwise stated in a credit line to the data. 
with dry eyes (keratoconjunctivitis sicca). She initially presented with "pins- and- needles" sensation (paresthesia) over the bilateral palms and feet, which developed simultaneously. These symptoms were followed by progressive weakness in the bilateral arms and legs distally more than proximally. This severely impaired her BADL and caused her to be wheelchair-bound.

After 10 days of progressive weakness in all four limbs, she exhibited poor muscle strength, which rendered her unable to stand and walk. She became completely wheelchair-dependent, which compelled her to visit our neurology clinic. The neurological examination revealed acute progressive weakness/paresthesia in all the four limbs, with generalized hyporeflexia. Nerve conduction study (NCS) of her bilateral upper and lower limbs revealed axonal sensorimotor polyneuropathy and severe bilateral distal median neuropathy at the wrists. Acute polyneuropathy was pronounced. We advised her to visit our emergency room (ER) for further examination, but she refused further clinical evaluation due to personal reasons. Her medical history indicated that she had been admitted to our Ophthalmology department from July 17-27, 2020, for the following reasons 1) corneal ulcer with corneal perforation in the right eye status after corneal suture and tissue glue, 2) purulent endophthalmitis in the right eye following intravitreal injection of antibiotics, and 3) superficial punctate keratitis (SPK) in the left eye.

On August 4, 2020, she was sent to our ER due to acute urinary retention and progressive weakness in all limbs. In the ER, the neurological examination revealed clear consciousness, incomplete closure of the left eyelid, no bulbar signs and symptoms, and quadriplegia with muscle strength scores as follows: upper limbs proximal2, distal-3 and lower limbs proximal-1, distal-2, as per the Medical Research Council Scale (MRC) scale, generalized absence of deep tendon reflexes, and paresthesia/ impaired joint position sense with glove-and-stocking distribution. Cervical and thoracic spine magnetic resonance imaging (MRI) revealed old compression fractures at T6-T7 and mildly hyperintense T2 lesion at the C3C4 level (Fig. 1).

Based on the initial clinical presentation, GuillainBarré syndrome (GBS) was suspected. Estimation of the levels of serum tumor markers, autoimmune profile tests, NCS of the bilateral upper and lower limbs, stool culture, orbital MRI with contrast, and lumbar puncture were performed to establish accurate diagnosis and exclude other possibilities, such as transverse myelitis and central nervous system infection.

Serum tumor markers and autoimmune profiles yielded high titers of anti-SSA and anti-SSB autoantibodies (Table 1). NCS of the bilateral upper and lower limbs revealed severe axonal sensorimotor polyneuropathy and severe bilateral distal median neuropathy at the wrists, with a lower amplitude than that in the previous study. Orbital MRI results were negative. Stool culture was negative for Campylobacter. Lumbar puncture consistently revealed albuminocytologic dissociation, compatible with suspected GBS. Based on the clinical manifestations, electrodiagnostic criteria [7], and cerebrospinal fluid analysis, we tentatively diagnosed the patient with AMSAN-variant GBS.

The patient did not consent to plasma exchange. Therefore, intravenous immunoglobulin (IVIG) treatment was considered. Meanwhile, intravenous methylprednisolone $(1000 \mathrm{mg} /$ day $)$ was prescribed on the third day of admission. The steroid pulse therapy lasted for 5 days, during which the left upper distal limb muscle strength score improved to MRC scale 3. Bilateral feet

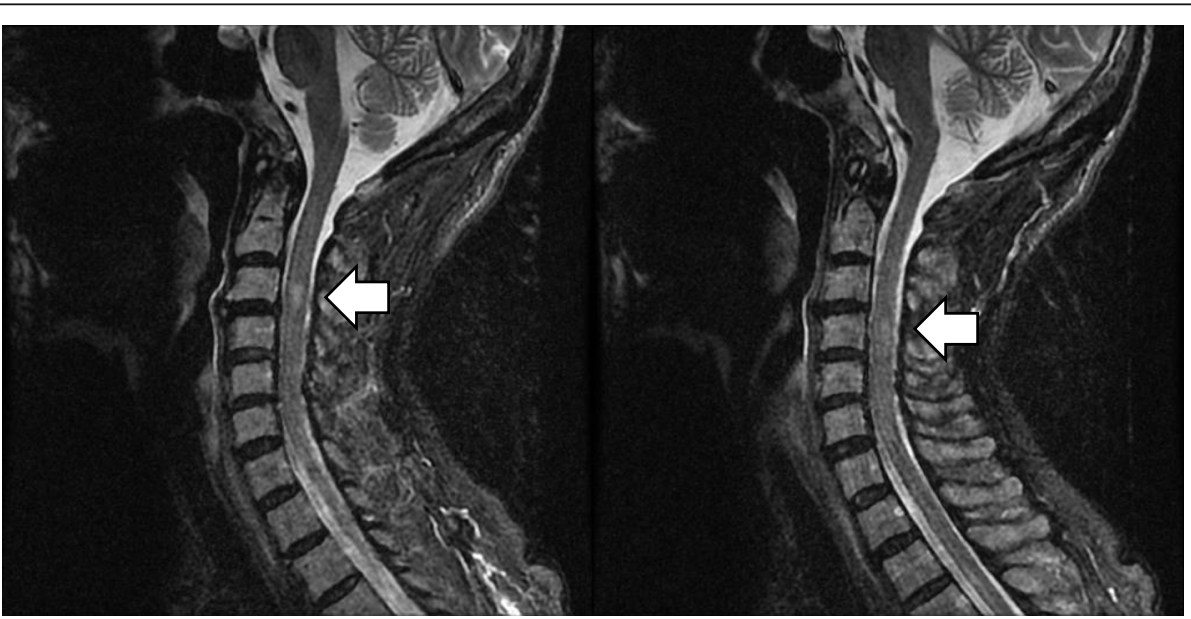

Fig. 1 T2 weighted cervical magnetic resonance imaging (MRI) without contrast. Cervical spine MRI showing mild increase in the T2-signal intensity of the cervical cord at the C3-C4 levels 
Table 1 Autoimmune serological test results

\begin{tabular}{|c|c|c|}
\hline Antibody & Values & $\begin{array}{l}\text { Normal } \\
\text { values }\end{array}$ \\
\hline Anti-nuclear antibody & 1:640 (+), homogenous & $<1: 40$ \\
\hline Anti-SSA (EliAU/mL) & $>240$ & 10 \\
\hline Anti-SSB (EliAU/mL) & 107 & 10 \\
\hline Anti-CENP (EliAU/mL) & 0.6 & 10 \\
\hline Anti-DNA (IU/mL) & 0.8 & 15 \\
\hline Anti-RF (IU/mL) & $<10$ & 14 \\
\hline Anti-TPO (IU/mL) & 0.6 & 9 \\
\hline Anti-TG (IU/mL) & $<0.9$ & 4 \\
\hline Lupus anticoagulant & 1.2 & 1.2 \\
\hline Anti-RNP (EliAU/mL) & 1.2 & 10 \\
\hline Anti-Smith (EliAU/mL) & 0.9 & 10 \\
\hline c-ANCA (IU/mL) & 0.2 & 3 \\
\hline p-ANCA (IU/mL) & 0.2 & 5 \\
\hline Anti-Cardiolipin-lgG (GPL U/mL) & $<1.6$ & 20 \\
\hline Anti-Cardiolipin-IgM (MPL U/mL) & 7.1 & 12.5 \\
\hline Anti-ß2GP1-lgG (U/mL) & $<1.4$ & 20 \\
\hline Anti-ß2GP1-IgM (U/mL) & 1.2 & 20 \\
\hline Anti-AQP4 & Negative & Negative \\
\hline
\end{tabular}

Serologic tests revealed positive anti-SS-A and anti-SS-B antibodies $A Q P 4$ aquaporin 4, $\beta 2 G P 1$-lgG beta2 glycoprotein IgG, $\beta 2 G P 1-\lg M$ beta2 glycoprotein IgM, CENP Centromere IgM, c-ANCA cytosolic anti-neutrophil cytoplasmic antibody, Cardio-IgG Cardiolipin IgG, Cardio-IgM Cardiolipin IgM, $p$ ANCA perinuclear anti-neutrophil cytoplasmic antibody, RF Rheumatoid factor, RNP Ribonuclear protein, SSA Sjögren's syndrome A, SSB Sjögren's syndrome B, TPO Thyroid peroxidase, TG Thyroglobulin

and right palm paresthesia ameliorated, and the right leg muscle strength score improved to MRC scale 3 over the ensuing days. A rheumatologist was consulted regarding the elevated serum levels of anti-SSA and antiSSB autoantibodies. According to the 2016 ACR/EULAR classification criteria for pSS [8], the patient had high titers of anti-SSA antibodies as well as corneal ulcers with severe SPK in the left eye as a surrogate of ocular staining score or van Bijsterveld score. Thus, the rheumatologist was able to diagnose pSS and also classify it.

We administered IVIG after steroid pulse therapy. There was a remarkable improvement in the proximal limb muscle strength on the third day of IVIG administration. Moreover, the patient's clinical status significantly improved over the next few days. Continuous improvement in muscle power in all four limbs proximally as well as distally was noted. The patient also regained the use of axial muscles. Therefore, the rehabilitation program was upgraded due to her progress; however, she refused further transfer to the rehabilitation department as she wanted to go home early. Finally, the patient was discharged after 37 days of admission with partially-dependent BADL.
During the follow-up at the neurology outpatient clinic 2 weeks after discharge, the patient's muscle strength progressively improved (upper proximal-3, distal-3, lower proximal-4, distal-3), and the incomplete left eye closure resolved.

\section{Discussion and conclusions}

pSS is a chronic, autoimmune disease that usually occurs in middle-aged adults (mean age of onset: 4th to 5th decade of life) and is predominant in females. Its clinical picture varies among affected individuals. The most common symptoms are xerophthalmia and xerostomia. According to the 2016 ACR/EULAR classification criteria [8], any individual who fulfills the inclusion criteria with a weighted summed score of $\geq 4$ can be diagnosed with pSS.

Most patients with pSS initially experience neurological symptoms, wherein both peripheral and central nervous systems could be affected [1-3]. These symptoms vary widely, including axonal sensory neuropathy, axonal sensorimotor neuropathy, GBS, small- fiber neuropathy, and demyelination $[5,6]$. If the sicca symptoms (xerophthalmia and xerostomia) are subclinical and vague, diagnosis of pSS may be delayed or underdiagnosed.

There are multiple hypotheses regarding the neurological symptoms associated with the pathogenicity of pSS [6], including small-vessel vasculopathy [9], dorsal root ganglionitis [10], cryoglobulinemia [11], demyelination [12], myelitis [13], and antibody-mediated autonomic dysfunction [14]. This heterogeneity of the underlying neuropathological mechanisms of pSS complicates the treatment approach.

AMSAN is a rare form of GBS variants. The pathology is predominantly axonal loss of both motor and sensory nerve fibers. Although AMSAN has characteristics similar to those of acute motor axonal neuropathy (AMAN), the onset of AMSAN is rapid and its symptoms are more severe, resulting in significant disability [15]. AMSAN and AMAN are associated with Campylobacter jejuni infection, which in itself is a poor prognostic factor [16].

It is possible that our patient coincidentally had both AMSAN and pSS, although there may be an association between them. After we excluded other causes of acute polyneuropathy, we concluded that AMSAN may be associated with pSS based on the autoimmune serologic data and ocular lesions. Notably, the subclinical cervical myelitis observed on cervical MRI may be regarded as an accidental finding of several neurological conditions associated with Sjögren's syndrome [1-3].

As mentioned in an earlier study, the first line of treatment for GBS is immunotherapy with immunoglobulin or plasma exchange, with no significant differences between the two with regard to the clinical outcomes [17]. Currently, there is no known evidence of the beneficial 
effects of corticosteroids in the treatment of this condition [18, 19]. A meta-analysis of six trials comprising 587 participants, including patients with GBS, revealed no significant improvement in the disability score of patients treated with glucocorticoids compared with those who were not treated with these drugs [20]. However, the best treatment for AMSAN is unknown as there are very few studies available on this topic in the literature.

Methylprednisolone pulse therapy was prescribed for our patient after she refused plasma exchange. Mild improvement with no worsening of weakness and paresthesia in the limbs was noted. Subsequently, we administered IVIG for definite immunosuppression. The clinical response of the patient to this treatment was excellent, and she demonstrated good antigravity muscle strength in her limbs and residual mild right palmar paresthesia upon discharge. Although the early use of immunosuppressive therapy helped achieve clinical improvement during the acute stage, it did not completely prevent AMSAN-related functional damage. However, during the follow-up, the AMSAN sequelae continued to improve.

In this report, we presented a case of AMSAN with pSS and demonstrated that limb weakness may be improved by immunosuppressive therapy. The notable points in our case report are as follows: (i) the neurological manifestations of pSS precede the sicca symptoms; (ii) the symptoms vary widely, from AMSAN to sub-acute myelitis; and (iii) early and aggressive immunosuppressive therapy resulted in a remarkable clinical response in the patient.

This case emphasizes the importance of comprehensive immunological testing for patients who present with variants of GBS.

\section{Abbreviations}

AMSAN: Acute motor and sensory axonal neuropathy; AMAN: Acute motor axonal neuropathy; BADL: Basic activities of daily living; ER: Emergency room; GBS: Guillain-Barré syndrome; IVIG: Intravenous immunoglobulin; MRC scale: Medical Research Council scale; MRI: Magnetic resonance imaging; NCS: Nerve conduction study; OD: Oculus dexter; OS: Oculus sinister; SSA: Sjögren's syndrome-related antigen A; SSB: Sjögren's syndrome-related antigen B; SPK: Superficial punctate keratitis

\section{Acknowledgments}

This manuscript was edited by Enago English Editing.

\section{Authors' contributions}

All authors were involved in the management of this patient. Writing-original draft: YMC. Writing-review and editing: YMC, KYS. All authors read and approved the final manuscript.

\section{Funding}

The authors declare that they have no sources of funding.

\section{Declarations}

Ethics approval and consent to participate

This study was approved by the Research Ethics Committee of Hualien Tzu Chi Hospital, Buddhist Tzu Chi Medical Foundation (CR110-01) on January/ $13 / 2021$.

\section{Consent for publication}

Written consent to publish this information was obtained from the study participant.

\section{Competing interests}

The authors declare that they have no competing interests.

\section{Author details}

${ }^{1}$ Department of Neurology, Hualien Tzu Chi Hospital, Buddhist Tzu Chi Medical Foundation, Hualien, Taiwan. ${ }^{2}$ School of Medicine, Tzu Chi University, Hualien, Taiwan. ${ }^{3}$ Division of Allergy, Immunology \& Rheumatology, Hualien Tzu Chi Hospital, Buddhist Tzu Chi Medical Foundation, Hualien, Taiwan.

Received: 19 January 2021 Accepted: 8 April 2021

Published online: 15 April 2021

\section{References}

1. McCoy SS, Baer AN. Neurological complications of Sjögren's syndrome: diagnosis and management. Curr Treatm Opt Rheumatol. 2017;3(4):275-88. https://doi.org/10.1007/s40674-017-0076-9.

2. Margaretten M. Neurologic manifestations of primary Sjögren syndrome. Rheum Dis Clin N Am. 2017;43(4):519-29. https://doi.org/10.1016/j.rdc.2017. 06.002 .

3. Bhattacharyya S, Helfgott SM. Neurologic complications of systemic lupus erythematosus, Sjögren syndrome, and rheumatoid arthritis. Semin Neurol. 2014;34(04):425-36. https://doi.org/10.1055/s-0034-1390391.

4. Mori K, lijima M, Koike H, Hattori N, Tanaka F, Watanabe H, et al. The wide spectrum of clinical manifestations in Sjögren's syndrome-associated neuropathy. Brain. 2005;128(Pt 11):2518-34. https://doi.org/10.1093/brain/a wh605.

5. Chai J, Logigian EL. Neurological manifestations of primary Sjögren's syndrome. Curr Opin Neurol. 2010;23(5):509-13. https://doi.org/10.1097/ WCO.0b013e32833de6ab.

6. Teixeira F, Moreira I, Silva AM, Vasconcelos C, Farinha F, Santos E. Neurological involvement in primary Sjögren syndrome. Acta Reumatol Port. 2013;38(1):29-36.

7. Uncini A, Kuwabara S. Electrodiagnostic criteria for Guillain-Barré syndrome: a critical revision and the need for an update. Clin Neurophysiol. 2012; 123(8):1487-95. https://doi.org/10.1016/j.clinph.2012.01.025.

8. Shiboski CH, Shiboski SC, Seror R, Criswell LA, Labetoulle M, Lietman TM, et al. 2016 American College of Rheumatology/European League Against Rheumatism Classification Criteria for Primary Sjögren's Syndrome: A consensus and data-driven methodology Involving three international patient cohorts. Arthritis Rheumatol. 2017;69:35-45. https://doi.org/10.1 002/art.39859.

9. Alexander EL. Neurologic disease in Sjögren's syndrome: mononuclear inflammatory vasculopathy affecting central/peripheral nervous system and muscle. A clinical review and update of immunopathogenesis. Rheum Dis Clin N Am. 1993;19(4):869-908.

10. Griffin JW, Cornblath DR, Alexander E, Campbell J, Low PA, Bird S, et al. Ataxic sensory neuropathy and dorsal root ganglionitis associated with Sjögren's syndrome. Ann Neurol. 1990;27(3):304-15. https://doi.org/10.1 002/ana.410270313.

11. Feldman L, Dhamne M, Li Y. Neurologic manifestations associated with cryoglobulinemia: a single center experience. J Neurol Sci. 2019;398:121-7. https://doi.org/10.1016/j.jns.2019.01.041.

12. Min JH, Kim HJ, Kim BJ, Lee KW, Sunwoo IN, Kim SM, et al. Brain abnormalities in Sjögren syndrome with recurrent CNS manifestations: association with neuromyelitis optica. Mult Scler. 2009;15(9):1069-76. https://doi.org/10.1177/1352458509106228.

13. Qiao L, Wang Q, Fei Y, Zhang W, Xu Y, Zhang Y, et al. The clinical characteristics of primary Sjögren's syndrome with neuromyelitis optica spectrum disorder in China: A STROBE-compliant article. Medicine. 2015;94: e1145. https://doi.org/10.1097/MD.0000000000001145. 
14. Mukaino A, Nakane S, Higuchi O, Nakamura H, Miyagi T, Shiroma K, et al. Insights from the ganglionic acetylcholine receptor autoantibodies in patients with Sjögren's syndrome. Mod Rheumatol. 2016;26(5):708-15. https://doi.org/10.3109/14397595.2016.1147404.

15. Dimachkie MM, Barohn RJ. Guillain-Barré syndrome and variants. Neurol Clin. 2013;31 (2):491-510. https://doi.org/10.1016/..ncl.2013.01.005.

16. Ho TW, Mishu B, Li CY, Gao CY, Cornblath DR, Griffin JW, et al. Guillain-Barré syndrome in northern China. Relationship to Campylobacter jejuni infection and anti-glycolipid antibodies. Brain. 1995;1 18(Pt 3):597-605. https://doi. org/10.1093/brain/118.3.597.

17. Hughes RA, Swan AV, van Doorn PA. Intravenous immunoglobulin for Guillain-Barré syndrome. Cochrane Database Syst Rev. 2014;2014:CD002063. https://doi.org/10.1002/14651858.CD002063.pub6.

18. Hughes RA. Ineffectiveness of high-dose intravenous methylprednisolone in Guillain-Barré syndrome. Lancet. 1991;338(8775):1142. https://doi.org/10.101 6/0140-673691995-7.

19. Guillain-Barré Syndrome Steroid Trial Group correspondence to Prof R. A. C. Hughes. Double-blind trial of intravenous methylprednisolone in GuillainBarré syndrome. Lancet. 1993;341:586-90.

20. Hughes RA, Brassington R, Gunn AA, van Doorn PA. Corticosteroids for Guillain-Barré syndrome. Cochrane Database Syst Rev. 2016;10:CD001446. https://doi.org/10.1002/14651858.CD001446.pub5.

\section{Publisher's Note}

Springer Nature remains neutral with regard to jurisdictional claims in published maps and institutional affiliations.

Ready to submit your research? Choose BMC and benefit from:

- fast, convenient online submission

- thorough peer review by experienced researchers in your field

- rapid publication on acceptance

- support for research data, including large and complex data types

- gold Open Access which fosters wider collaboration and increased citations

- maximum visibility for your research: over $100 \mathrm{M}$ website views per year

At BMC, research is always in progress.

Learn more biomedcentral.com/submissions 